\title{
Rhomboid Concave and Convex Dimples Applied for Cooling in Gas Turbines
}

\author{
Galina Ilieva \\ University of Beira Interior, 6201-001 Covilhã, Portugal
}

\begin{abstract}
The scope of this work is to contribute towards a more efficient cooling design platforms for gas turbines. Concave and convex schemes of rhomboid dimples, arranged irregularly in a cooling channel, are numerically modeled and studied for their effects on heat transfer and cooling of turbine blades. Research is fulfilled in Gambit and Fluent based on a mathematical model and specified boundary conditions for modeling of aero- and thermodynamic characteristics of flow in cooling ducts. The proposed design shape and arrangement of dimples is found to be very promising and effective for intensive heat transfer and cooling in gas turbines and other industrial applications.
\end{abstract}

Keywords: Aerothermodynamics, Blade cooling, Dimples, Gas turbine, Heat transfer, Secondary flow

\section{Introduction}

Turbines are widely applied in marine propulsion, aircraft propulsion, power plants and other industrial areas. In recent years, the power requirements are significantly increasing, leading to the need of enhanced power, delivered by the turbine, and higher thermal efficiency. A very high inlet temperature of the flue gases entering the turbine stages is a prerequisite to increased power output and high efficiency performance. However, in gas turbines, high inlet temperature of flue gases could be damaging for the blade material. Therefore, it is very important to assure adequate cooling for both stator and rotor blades to avoid material failures.

Engineers must accurately study, model and analyze the aerothermodynamic features of flow past turbine blades to predict the local heat transfer coefficients, places of very high temperatures and thermal peaks, in order to introduce appropriate innovative cooling techniques to increase the life time of blades.
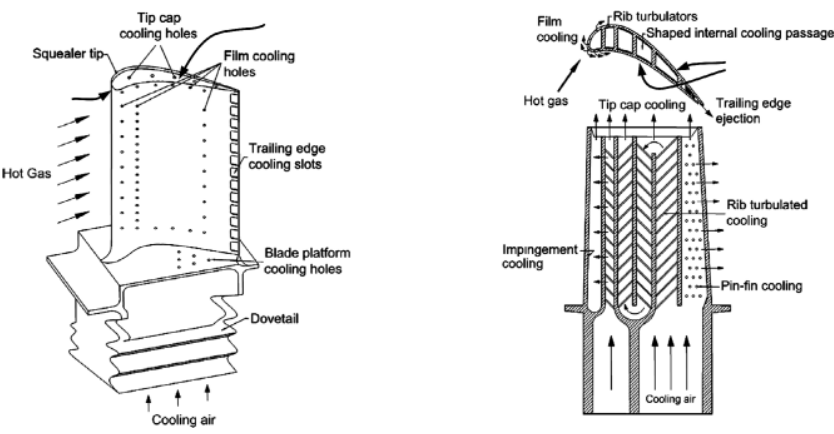

Figure 1: Schematic of a modern gas turbine blade with cooling [1]

Over the last 5 decades, there are many research works concerned on gas turbine blade cooling techniques. Analytical, computational and experimental methods are applied to study and improve various cooling approaches in gas turbines [2].
Many techniques have been developed to increase the rate of heat transfer and obtain cooling effect. Some of them have also been combined to achieve further increase in heat transfer rates.

Heat heat transfer effects and specifics, various cooling techniques are discussed in details in [3,4].

One of the well-known methods to improve the heat transfer (i.e., enhance material cooling) in a flow passage is to apply on the streamed internal blade surfaces turbulators (also they are called ribs), so that the surface area increases and significantly improves the cooling process. [5]. However, the application of rib turbulators poses some negative effects as decrease in the pressure of the coolant air, etc.

Cooling passages and their possible optimization are researched by Kim and Kim [6], they work on optimization of internal cooling passages with straight rectangular ribs [7], V-shaped ribs [8] and the angle of the ribs [9]. Values of geometric design variables are identified, also it is discussed that application of a numerical approach is a reliable way for optimization of heat transfer surfaces.

Internal cooling is carried out by fluid, called coolant, flowing through a number of serpentine passages, placed inside the blades and extracting heat from blade walls, fig. 1 . At the leading edge of the stator blade (at the flow stagnation point and in a close proximity to it), the heat transfer coefficients have very high values. After the leading edge, the flow is streaming along the concave and convex sides of the blade and heat fluxes will decrease. As it is known from the aerodynamics of a turbine blade, along its suction side, a transition phenomenon is observed and the flow becomes turbulent; values of heat transfer coefficients increase. Along the pressure side, the flow accelerates and heat coefficients also will increase.

The heat transfer in blade passages is affected by high turbulence rates, transition effects, surface roughness and secondary flows in inter-blade channels, also by rotational effects and forces, leakage in axial and radial gaps. 


\section{International Journal of Science and Research (IJSR) \\ ISSN (Online): 2319-7064 \\ Index Copernicus Value (2013): 6.14 | Impact Factor (2014): 5.611}

There are various approaches to perform cooling in passages in gas turbines [14]. Most of them are described in this paragraph.

\subsection{Rib Turbulated Cooling}

Rib turbulators are used to increase the heat transfer in the internal cooling passages designed as serpentines. The rib turbulators are cast on two opposite walls of the cooling passage, fig. 2. The heat transfer in case of applied rip turbulators depends on the shape and dimensions of the rib turbulators, channel aspect ratio, where ribs are placed along the channels, Reynolds number of the flow, etc. Many research works have been accomplished to understand the coolant flow aero- and thermodynamics through a stationary ribbed channel [4,510,11,16].

It is observed that when the flow used as cooler passes over a rib oriented at angle of $90^{\circ}$ to the main stream, the flow that is streaming walls, separates. Separation is followed by reattachment as the reattached boundary layer results in increased heat transfer coefficients over the surfaces of the ribbed channel. Ribs also induce secondary flow as it is shown in fig. 2. If the rib turbulators are skewed and oriented to the mainstream flow direction, counter-rotating vortices are created. A V-shaped rib turbulator creates 4 counterrotating vortices and results in more intensive heat transfer enhancement in the channel. Ribs create turbulent mixing in the areas of flow separation and contribute to highly effective heat transfer.

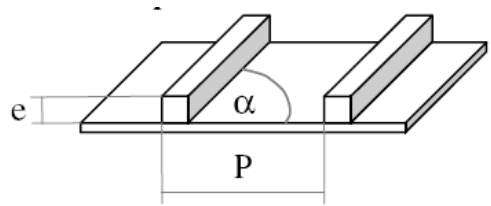

Flow field between successive ribs

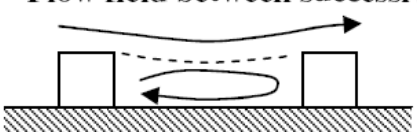

Figure 2: Ribs inducing secondary flows [15].

Skewed ribs (placed at angle to the main stream) contribute to enhanced heat transfer. Ribs, placed at angle of attack in the range $30^{\circ}-60^{\circ}$, also lead to increased heat transfer and reduced pressure drag. The height of the ribs is $5-10 \%$ of the channel hydraulic diameter, and the rib spacing-to-height ratio varies from 5 to 15 [14]

In $[11,16]$ is demonstrated that $\mathrm{V}$-shaped ribs are better than the angled ribs; for a given pressure drop, the $\mathrm{V}$-shaped ribs give more heat transfer enhancement. In fig. 3 are shown the so called broken ribs - they are similar to the traditional ones, but the rib is not continuous. In the majority of cooling channels, discrete ribs have shown better behavior than the continuous angled or V-shaped ribs, Cho et al. [12].

\subsection{Impingement Cooling}

Impingement cooling is applied to increase the heat transfer rates and decrease thermal loads along the leading edge of turbine blades. Impingement cooling is found to be appropriate for application near the mid-chord of the inlet guide vanes. Sharp curvature at the leading edge of the vane must be considered when utilizing impingement in this region. In fig. 4 is presented an example of impingement cooling scheme used by for research in [13].

Detailed information regarding the jet impingement from multiple jets, jet impingement on a curved surface, among others, as better approaches for cooling is discussed in [14]

In [17] is concluded that for multiple jets, the Nusselt number is strongly dependent on the Reynolds number. In [17] is discussed that the mass from one jet moves in the cross-jet flow direction, and this flow can alter the performance of neighboring jets [5]. Correlations based on experimental data were developed and presented in $[16,18]$ to acquire the heat transfer rates from an array of impinging jets. In [19] is studied impingement on a curved surface, and this group concluded that the average Nusselt number ratio increases as the curvature of the experimental plate increases.

\subsection{Pin-Fin Cooling}

Pin-Fin cooling is applied due to various manufacturing obstacles in the very narrow part of the trailing edge. In a pin-fin array heat is transferred from the smooth channel endwall and from the pins. As the coolant flows past the pin, the flow separates and wakes are shed downstream of the pin. In addition to this wake formation, a horseshoe vortex forms just upstream of the base of the pin, and the vortex wraps around the pins. This horseshoe vortex creates additional mixing, and thus enhanced heat transfer [14].

Many factors must be considered when investigating pin-fin cooling. The type of pin-fin array and the spacing of the pins in the array effect the heat transfer distribution in the channel. The pin size and shape also have a profound impact on the heat transfer in the cooling passage [14].

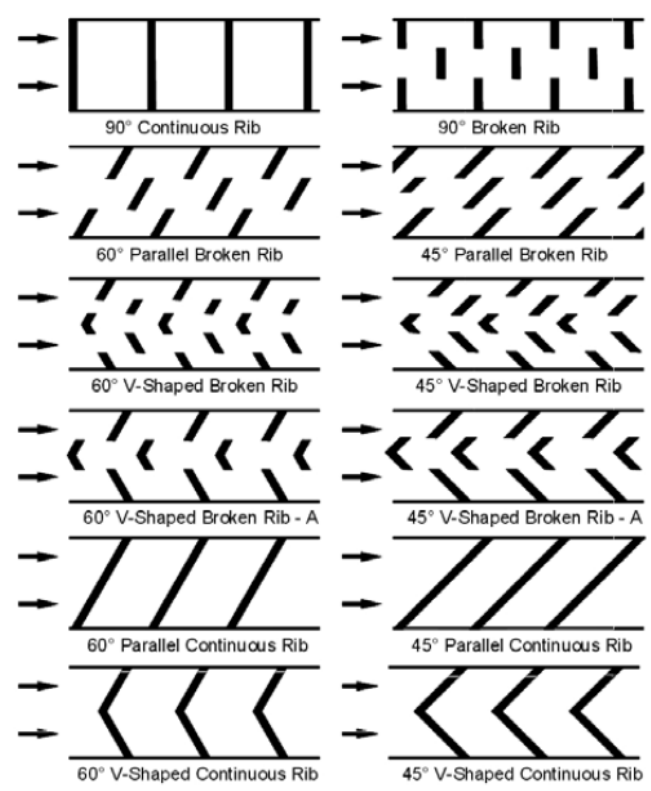

Figure 3: High performance rib turbulators [14] 


\section{International Journal of Science and Research (IJSR) \\ ISSN (Online): 2319-7064 \\ Index Copernicus Value (2013): 6.14 | Impact Factor (2014): 5.611}

In [21] is studied the effects of partial length pins in a rectangular channel.

Chyu et al. used cube and diamond shaped pins to enhance the heat transfer coefficient from a surface [22]. The cubeshaped pins have the highest mass transfer coefficients among the shapes considered and round pins have the lowest mass transfer coefficients.

\subsection{Dimple Cooling}

Dimples, shown in Figure 6, are applied as an alternative of the pin-fin cooling. Dimpled cooling is characterized with relatively low pressure drag and high heat transfer levels. The concave dimples induce flow separation and reattachment with pairs of vortices. The areas of high heat transfer include the areas of flow reattachment on the flat surface immediately downstream of the dimple. The heat transfer in the dimpled channel is typically 2 to 2.5 times greater than the heat transfer in a smooth channel with loss due to a pressure drag of 2 to 4 times that of a smooth channel.

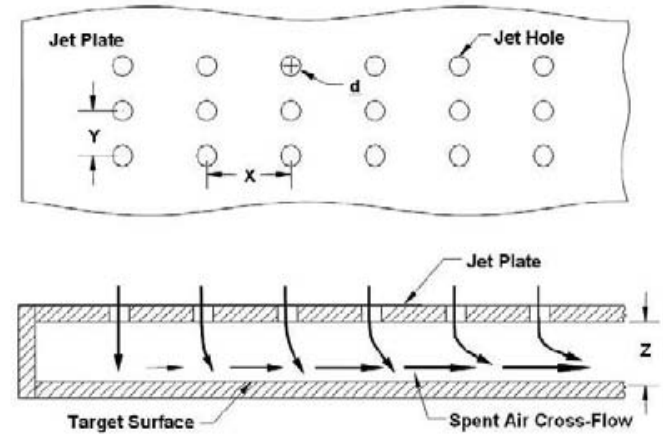

Figure 4: Impingement cooling for Turbine Blade Internal Cooling [14]

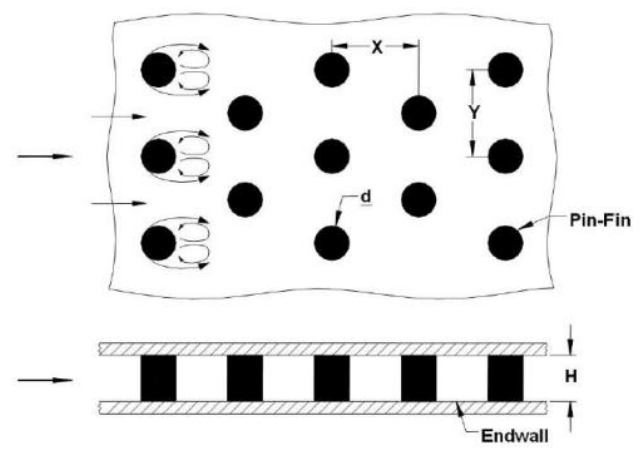

Figure 5: Pin-Fin cooling with detailed picture of vortices in a concave dimple [14]

An introduction of several new cooling techniques, their application, and specifics is presented in details in [14]. The literature survey shows that there are not many research activities and publications related to different than the aforementioned geometries for turbulators in cooling channels.

The overall goal of this research is to propose and investigate geometry and irregular distribution of rhomboid dimples, concave and convex, targeted to cooling optimization in gas turbine blades.
Dimple parameters:

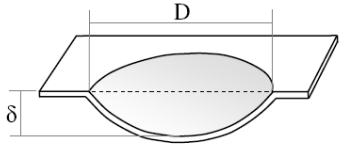

Dimple pattern:

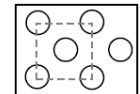

square

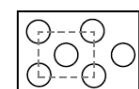

rectangular

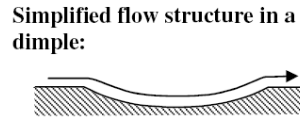

$\delta / \mathrm{D}<0.1$ (shallow dimple)

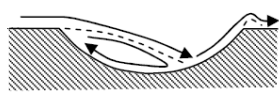

$\delta / \mathrm{D}>0.1$ (deep dimple)
Figure 6: Dimples as turbulators in cooling channels, [15].

\section{Problem set-up}

\subsection{Geometry and numerical modeling}

The purpose of current research is 3D modeling and analysis of a set of dimples having rhomboid shape, arranged in a specific way in the cooling channel. The idea of such design is to attain intensive vortex structures (secondary flows), produce higher rates of heat transfer and intensive cooling of high temperature loaded surfaces.

Geometry modeling of both channels is fulfilled in Gambit, with discretization grid, refined around streamed walls. The total number of elements for both cases is around 198000 , fig. 7 .

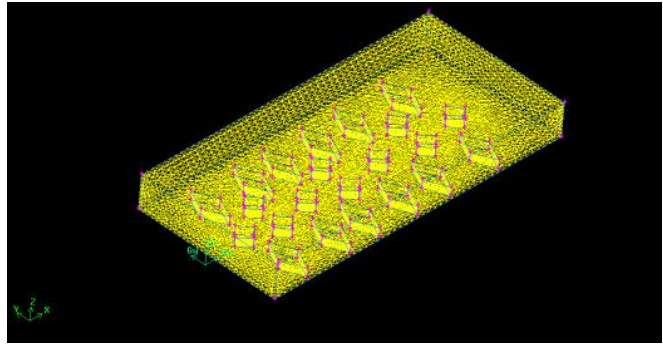

Figure 7: Discretized flow field.

Constant mass flow rate of coolant air is imposed in the flow direction. Mean bulk temperature is $300 \mathrm{~K}$. No-slip boundary conditions "wall" is implemented over the channel walls as well as over the walls of the ribs. The wall with distributed dimples is assumed as heated surfaces with constant heat flux of $1000 \mathrm{~W} / \mathrm{m} 2$, the other walls are imposed adiabatic. Cooling air enters through a boundary of type "mass-flow inlet".

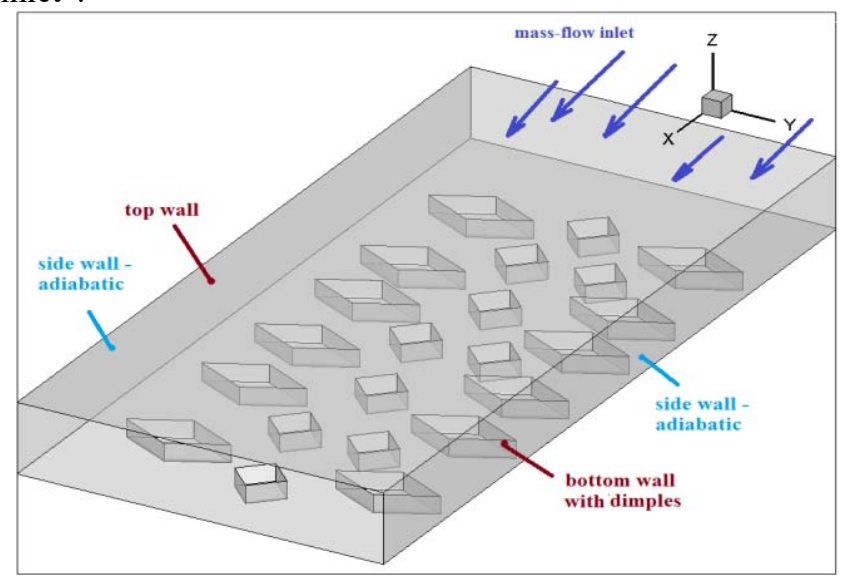

Figure 8: Domain configuration with imposed boundary zones. 


\section{International Journal of Science and Research (IJSR) \\ ISSN (Online): 2319-7064 \\ Index Copernicus Value (2013): 6.14 | Impact Factor (2014): 5.611}

Numerical model related to study the aerothermodynamics of a coolant in a channel, representing the serpentine installed in a gas turbine blade cooling space, is developed for steady 3D flow, periodic, fully developed, turbulent and viscous fluid air.

\subsection{Mathematical Model}

Reynolds averaged Navier-Stokes (RANS) equations are applied to acquire the aerothermodynamics of the flow field. RANS equations are as follows:

- continuity equation:

$$
\frac{\partial}{\partial x_{i}}\left(\rho u_{i}\right)=0
$$

-momentum equation:

$$
\frac{\partial}{\partial x_{j}}\left(\rho u_{i} u_{j}\right)=\frac{\partial p}{\partial x_{i}}+\frac{\partial}{\partial x_{j}}\left[\mu\left(\frac{\partial u_{i}}{\partial x_{j}}-\rho \overline{u_{i}^{\prime} u_{j}^{\prime}}\right)\right]
$$

-energy equation:

$$
\frac{\partial}{\partial x_{i}}\left(\rho u_{i} T\right)=\frac{\partial}{\partial x_{j}}\left[\left(\Gamma+\Gamma_{t}\right) \frac{\partial T}{\partial x_{j}}\right]
$$

where $\Gamma$ and $\Gamma$ tare molecular thermal diffusivity and turbulent thermal diffusivity, respectively and equal to

$$
\Gamma=\mu / \operatorname{Pr}, \Gamma_{t}=\mu_{t} / \operatorname{Pr}_{t}
$$

As a turbulence closure the Renormalization group (RNG) $k$ $\varepsilon$ model is applied [23].

This model predicts the overall heat transfer rate and gives good agreement with the experiments, [24].

The governing equations are discretized by the Second Order Upwind scheme, exception is the pressure-velocity coupling equation, discretized by SIMPLE scheme. Solutions is obtained with imposed values for all convergence factors equal to $10^{-6}$, exception is the energy equation with factor of convergence $10^{-8}$. Thermal performance is presented by heat transfer coeffcienct $\alpha$ and Nusselt number $\mathrm{Nu}$, as follows:

$$
\begin{aligned}
& \alpha=q /\left(T s-T_{B}\right) \\
& N u=\alpha \cdot D / k
\end{aligned}
$$

In last two equations Ts is the channel wall temperature, $T_{B}$ is the bulk temperature of fluid, $\mathrm{q}$ is the heat flux and $\mathrm{k}$ is the thermal conductivity, $\mathrm{D}$ is the hydraulic diameter of the channel, imposed in the panel related to turbulence boundary conditions.

\section{Results}

For the purposes of the results visualization, several crosssections along $\mathrm{x}, \mathrm{y}$, and $\mathrm{z}$ axis of the flow domain, were fulfilled. As it is obvious (fig. 8) dimples are not regularly distributed, but have a specific configuration. The idea behind that is: design and placement oriented to intensive flow turbulization, in order to empower the heat transfer and reduce temperature loading of blade surfaces. At places where dimples are concentrated, higher levels of secondary flows and rapid changes in flow parameters are observed.
Last mentioned, is suitable for places, in gas turbine ducts, where "hot spots" were identified to arise.

\subsection{Results for convex rhomboid dimples}

The heat transfer augmentation peak is more intense in the case of convex dimples. Also the overall local heat transfer levels are higher, which leads to a higher increase in the areaaveraged heat transfer rates.

Close to the channel walls, it is noticeable that there are regions of low turbulence intensity, lower vortices intensity, smaller pressure drops, see fig. 10(a), and heat transfer levels. Higher values are observed for the mid area along the channel, due to the many obstacles in front the incoming stream of coolant, leading to increased velocities (fig. 10(b) and decrease in pressure and temperature values, fig. 10(a). This type of distribution is appropriate for cases with indentified "hot spots" over blade surfaces. For example, that kind of cooling architecture may be placed at the region of the leading edge or to the concave blade surfaces or where high thermal loads are measured.

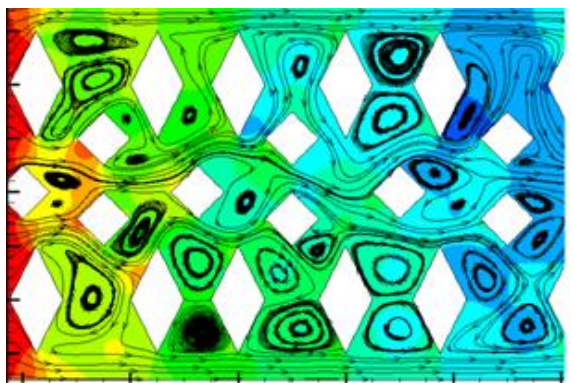

(a)

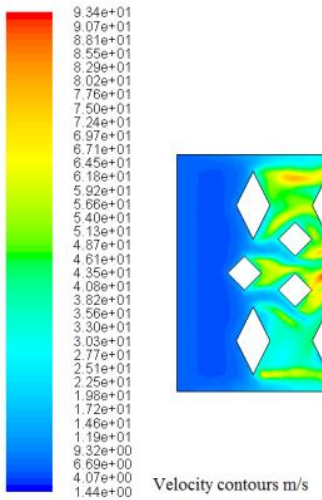

(b)

Figure 10 (a,b): Pressure flow field (a - zoomed in area) with secondary flows and velocity distribution (b) in a section realized through the rhomboid turbulators ( $\mathrm{z}=$ const)

\subsection{Results for concave rhomboid dimples}

Gas turbine blade cooling with application of concave dimples is characterized with lower pressure values (fig. 11(a) and velocities (fig. 11(b) in a close proximity to the dimples. As it is shown in fig. 11 and fig. 12, secondary flows and less vorticity are obtained only in the volume of the concave dimples and very close to them, fig. 12a. Also, these kind of cooling elements are characterized with relatively low pressure drag and high heat transfer levels. In fig $12(a, b)$ is presented the induced flow separation and 


\section{International Journal of Science and Research (IJSR) \\ ISSN (Online): 2319-7064 \\ Index Copernicus Value (2013): 6.14 | Impact Factor (2014): 5.611}

reattachment with pairs of vortices. The areas of high heat transfer include the areas of flow reattachment on the walls downstream of the dimples.

Skin friction coefficient in channel with convex dimples varies in the range 0.00802 to 0.281 . In channel with concave dimples values for skin friction coefficient are 0.00164 to 0.197 . This is due to the less level of secondary flows and ortex structures.

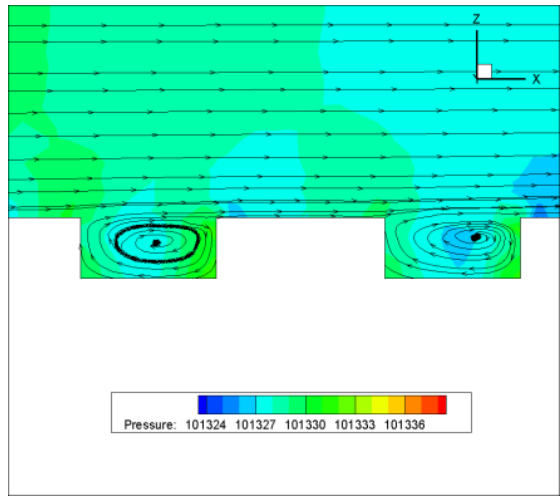

(a)
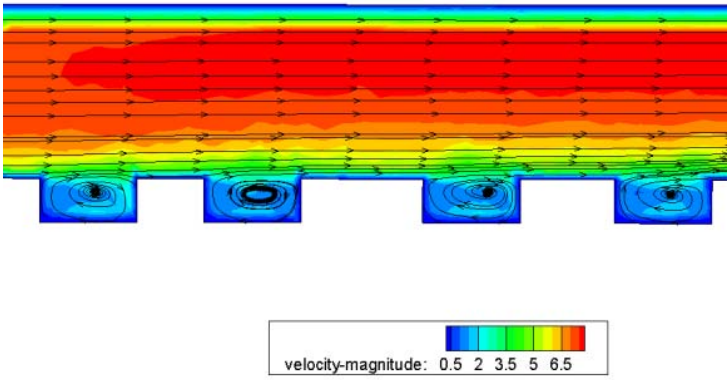

(b)
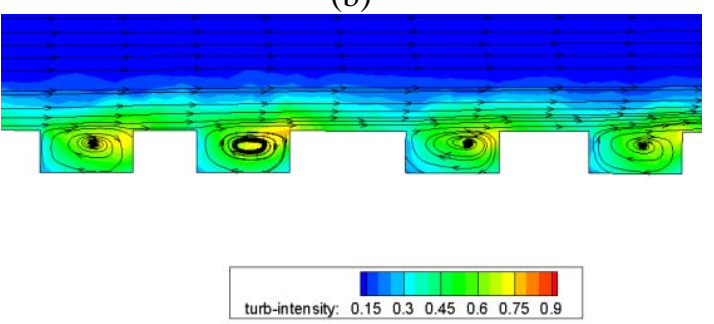

(c)

Figure 11 (a,b,c): Pressure flow (a), velocity flow (b) and turbulent intensity (c) in a zoomed in area with vortex structures in dimples, in a section along the channel ( $\mathrm{y}=$ const).

Maximum value of surface Nusselt number for the first case of research is 962, for the second case with implemented concave dimples is 1280 .

After calculation of heat transfer it is found that heat transfer in the dimpled channel with concave elements is 1.98 times greater than the one in a smooth channel (results for smooth channel are not shown here), but there are losses due to pressure drag over streamed walls. In the same time, a comparison between convex and concave dimples shows that the convex ones produce more vortical structures and high pressure drag values.
It could be concluded that both approaches for cooling have their great positive outcomes, instead of the fact that they go together with pressure drag and losses. A compilation between the both kinds of turbulators, with some changes in their geometry and variation in arrangement could be very promising solution for highly efficient cooling of gas turbines.

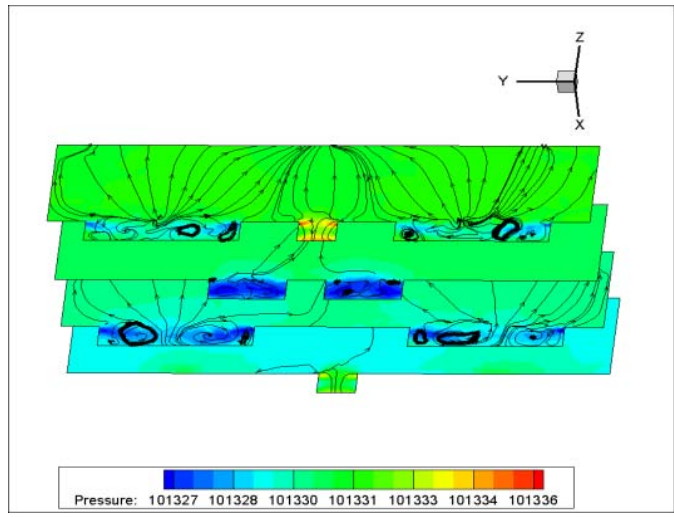

(a)

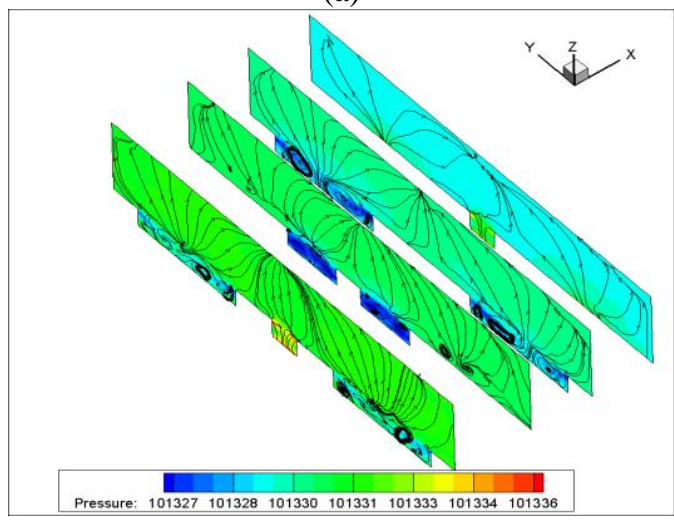

(b)

Figure 12: Pressure flow field and secondary flows in a sections realized along the cooling channel $(x=c o n s t)(a, b-$ various viewpoints)

\section{Conclusions}

The overall goal of this research was to propose, model and analyze different geometry and way of arrangement of turbulators targeted to cooling optimization in gas turbine blades.

After intensive modeling and research is found that the idea for application of rhomboid dimples (concave and convex), distributed in a specific way along a channel, is very promising for application in cooling ducts.

A dimpled channel with convex or concave elements leads to significantly high levels of heat transfer and cooling effect. Developed numerical methodology for research is widely applicable to study more innovative ideas for cooling in various industrial areas.

\section{References}

[1] Han JC. 2004. Recent studies in turbine blade cooling. International Journal of Rotating Machinery, 10(6), 443457. 


\section{International Journal of Science and Research (IJSR) \\ ISSN (Online): 2319-7064}

Index Copernicus Value (2013): 6.14 | Impact Factor (2014): 5.611

[2] Nagaiah NR \& Geiger CD: Evolutionary numerical simulation approach for design optimization of gas turbine blade cooling channels. Int. J. Simul. Multisci. Des. Optim., 2014, 5, A22.

[3] R.J. Goldstein, "Heat Transfer in Gas Turbine Systems,"Annuals of The New York Academy of Sciences, New York,New York, 2001, Vol. 934,520 pages.

[4] J.C. Han, L.R. Glicksman, W.M. Rohsenow“, An Investigation ofHeat Transfer and Friction for RibRoughenedSurfaces,'International Journal of Heat and Mass Transfer 1978 21: 1143-1156.

[5] J.C. Han, J.S. Park, "Developing Heat Transfer in Rectangular Channels with Rib Turbulators,'International Journal of Heat and Mass Transfer 1988, 31: 183-195.

[6] Kim KY, Kim HM., "Shape optimization of ribroughened surface to enhance turbulent heat transfer", International Journal of Heat \& Mass Transfer, 2002, 45, 2719-2727.

[7] Kim HM, Kim KY. 2004. Design optimization of ribroughened channel to enhance turbulent heat transfer", International Journal of Heat and Mass Transfer, 2004, 47, 5159-5168.

[8] Kim KY, Lee YM., Design optimization of internal cooling passage with V-shaped ribs. Numerical Heat Transfer: Part A: Applications, 2004, 51, 11031118.

[9] Kim KY, Kim HM., Optimization of the three dimensional angled ribs with RANS analysis of turbulent heat transfer. Proceedings from the ASME Turbo Expo: Vienna, Austria, 2004.

[10] J.C. Han, J.S. Park, C.K. Lei,1985,"Heat Transfer Enhancement in Channels with Turbulence Promoters", ASME Journal of Engineering for Gas Turbines and Power 107: 628-635.

[11] J.C. Han, 1988, "Heat Transfer and Friction Characteristics in Rectangular Channels with Rib Turbulators", ASME Journal of Heat Transfer 110: 321328.

[12]H.H. Cho, S.J. Wu, H.J. Kwon, "Local Heat/Mass Transfer Measurements in a Rectangular Duct with Discrete Ribs", ASME Journal of Turbomachinery 2000, 122: 579-586.

[13] L.W. Florschuetz C.C. Su, "Effects of Crossflow Temperature on Heat Transfer Within an Array of Impinging Jets", ASME Journal of Heat Transfer, 1987, 109: 74-82.

[14] turbines/handbook/4-2-2-2.pdf, cited 02.09.2015

[15] Michael Maurer, Experimental and numerical investigations of convective cooling configurations for gas turbine combustors, Ph.D. Thesis, Stuttgart , Germany2008

[16] J.C. Han, S. Dutta, S.V. Ekkad, 2000, "Gas Turbine Heat Transfer and Cooling Technology", Taylor \& Francis, Inc., NewYork, New York, ISBN \# 1-56032841-X, 646 pages.

[17] Florschuetz, C.R. Truman, D.E. Metzger, 1981, "Streamwise Flow and Heat Transfer Distributions for Jet Array Impingement with Crossflow", ASME Journal of Heat Transfer 1989, 103: 337-342.
[18]D.E. Metzger, L.W. Florschuetz, D.I. Takeuchi, R.D. Behee, and R.A. Berry, "Heat Transfer Characteristics for Inlineand Staggered Arrays of Circular Jets with Crossflow of Spent Air", ASME

[19] D.M.Kercher, W. Tabakoff, "Heat Transfer by a Square Array of Round Air Jets Impinging Perpendicular to aFlat Surface Including the Effect of Spent Air,"ASME Journal of Engineering for Power, 1970, Vol. 92 : 73-82.

[20] R.E. Chupp, H.E. Helms, P.W. McFadden, and T.R.Brown, "Evaluation of Internal Heat Transfer Coefficients for Impingement Cooled Turbine Airfoils", AIAA Journal of Aircraft 1969 (6): pp.203-208.

[21] J.C. Bailey, R.S. Bunker, "Local Heat Transfer and Flow Distributions for Impinging Jet Arrays of Dense andSparse Extent", ASME Paper No. GT-2002-30473.

[22] S.C. Arora and W. Abdel-Messeh,"Characteristics of Partial Length Circular Pin Fins as Heat Transfer Augmentors for Airfoil Internal Cooling Passages", ASME Paper No. 89-GT-87, 1989.

[23] M.K. Chyu, Y.C. Hsing, T.I.P. Shih, V. Natarajan, 1998,"Heat Transfer Contributions of Pins and Endwall in Pin-FinArrays: Effects of Thermal Boundary Condition Modeling”, ASME Paper No. 98-GT-175.

[24] www.ansys.com, cited 12.10.2015.

[25] Khamaj J, An experimental study of heat transfer in the cooling channels of gas turbine rotor blades, Ph.D. Thesis, University Wales, UK. 